\title{
Legionella pneumophila Serogroup 5
}

National Cancer Institute

\section{Source}

National Cancer Institute. Legionella pneumophila Serogroup 5. NCI Thesaurus. Code C123502.

Subgroup 5 of Legionella pneumophila, differentiated by its antigenicity. 